\title{
SOBRE EL CONCEPTO DE ORDEN JURIDICO*
}

\author{
CARLOS E. ALCHOURRÓN \\ EUGENIO BULYGIN \\ Universidad de Buenos Aires
}

La idea de que las normas jurídicas no se presentan aisladas, sino formando parte de un conjunto más vasto, llamado orden jurídico, está muy difundida entre los juristas y es ampliamente aceptada por ellos. Kelsen la formula muy claramente al comienzo de su Teoría General del Derecho y del Estado:

El derecho es un orden de la conducta humana. Un "orden" es un conjunto de normas. El derecho no es, como a veces se dice, una norma. Es un conjunto de normas que tienen el tipo de unidad a que nos referimos cuando hablamos de un sistema. Es imposible captar la naturaleza del derecho si limitamos nuestra atención a una sola norma aislada. Las relaciones entre las normas de un orden jurídico son también esenciales a la naturaleza del derecho. Únicamente sobre la base de una clara comprensión de las relaciones que constituyen un ordenamiento jurídico, puede entenderse plenamente la naturaleza del derecho. ${ }^{1}$

* El presente trabajo resume en forma simplificada algunos de los resultados de las investigaciones efectuadas por los autores en el tema de la dero. gación. La limitación principal resulta de no considerar sino las normas dictadas por una autoridad, es decir, normas legisladas (en un sentido amplio) y dejar de lado las normas originadas en la costumbre. Con la inclusión de estas últimas resultaría insuficiente la división de las normas en formuladas y derivadas (ya que las normas consuetudinarias, siendo originarias, carecen de formulación canónica) y aumentaría considerablemente el alcance de la indeterminación lógica resultante de la derogación de las normas derivadas. Pero las conclusiones principales de este trabajo permanecerían vigentes, adquiriendo incluso un acento más dramático.

1 Hans Kelsen, Teoría General del Derecho y del Estado, 2a. ediciỏn (México, 1958), p. 3 . 
En consecuencia, los juristas han dedicado no pocos esfuerzos a la elucidación del concepto de orden jurídico, que constituye tradicionalmente uno de los temas centrales de la teoría general del derecho. Los resultados alcanzados no guardan, empero, relación con los esfuerzos realizados; las definiciones conocidas no van mucho más allá de la noción intuitiva que los juristas manejan en forma harto oscura. A continuación trataremos de analizar el concepto de orden jurídico, partiendo de la noción intuitiva aceptada y usada por los juristas.

Previamente, cabe hacer dos observaciones preliminares. En primer lugar, en lo que sigue hablaremos acerca de órdenes de normas y no de proposiciones normativas, es decir, proposiciones acerca de las normas. Esta aclaración tiene su importancia, puesto que respecto de este punto no hay claridad total - para decirlo suavemente- entre los autores. Si bien los juristas entienden, por lo común, cuando hablan de un orden jurídico positivo, v. gr. el derecho francés o boliviano, el conjunto de todas las normas jurídicas que pertenecen a dicho derecho, algunos filósofos prefieren hablar de orden o sistema de proposiciones normativas, posiblemente por advertir que las normas en su carácter de prescripciones no pueden ser verdaderas ni falsas y creyendo que tal circunstancia impide que haya entre ellas relaciones lógicas de deducibilidad o contradicción, necesarias para distinguir un orden sistemático (y se pretende que el derecho lo sea) de un mero conglomerado o agregado de objetos yuxtapuestos. Otros autores hablan indistintamente de sistemas de normas o sistemas de proposiciones normativas, confiando aparentemente en una suerte de armonía preestablecida que aseguraría un isomorfismo entre unas y otras. ${ }^{2}$ Sea cual fuere la situación respecto de las proposiciones de la ciencia jurídica,

2 Cfr. Roberto J. Vernengo, Curso de Teoria General del Derecho (Buenos Aires, 1972), cap. 9, pp. 267 ss. 
a nosotros nos interesa elucidar aquí el concepto de orden jurídico como conjunto de normas.

En segundo lugar, una aclaración terminológica. Al decir que el orden es un conjunto de normas no pretendemos afirmar que todos los enunciados que integran un orden jurídico sean normativos, aunque algunos de ellos han de serlo necesariamente. ${ }^{3}$ Por lo tanto, la expresión "norma jurídica" ha de entenderse en este contexto en un sentido lato (que con. cuerda con el uso corriente entre los juristas) para abarcar cualquier enunciado que figure en los textos que registran el derecho positivo - constituciones, códigos, leyes, etc.—, sea éste normativo o no.

\section{La noción intuitiva de orden jurídico}

Las ideas acerca del orden jurídico difundidas entre los juristas pueden resumirse en las ocho tesis siguientes:

I. El orden jurídico es la totalidad de las normas válidas de acuerdo a un criterio de validez.

II. El orden jurídico es un conjunto de normas.

III. El orden jurídico es un sistema de normas.

IV. Las normas jurídicas son creadas y aniquiladas por medio de actos de voluntad.

V. El orden jurídico es dinámico, es decir, está sujeto a cambios en el tiempo.

VI. Los cambios del orden jurídico afectan su contenido, pero no su identidad, es decir, el orden jurídico puede perdurar en el tiempo sin perder su identidad, aun cuando su contenido sea diferente en cada momento.

3 Cfr. C. E. Alchourrón y E. Bulygin, Introducción a la Metodología de las Ciencias Juridicas y Sociales (en adelante, Metodologia) (Buenos Aires, 1975), donde se usa un término menos comprometido "enunciado jurídico" o "enunciado de derecho"; ver pp. 111 y 115. 
VII. El contenido del orden jurídico es en todo momento determinado o al menos determinable, es decir, se puede determinar en principio qué normas pertenecen a un orden jurídico dado en un momento dado.

VIII. En todo Estado hay uno, y solamente un orden ju. rídico.

La mayoría de estas tesis son intuitivamente muy plausibles y todas están representadas en la literatura especializada. Pero no es fácil unirlas en una teoría coherente, pues las tesis transcritas son - por aceptable que parezca cada una de ellas - contradictorias. Algunas contradicciones pueden tal vez disolverse por medio de distinciones conceptuales adecuadas; otras son más tenaces y no se dejan eliminar fácilmente. Se va a ver más adelante que las tesis III y IV son incompatibles con VII y probablemente también con VIII.

La tesis I puede ser considerada como una definición (incompleta) de un concepto de orden jurídico y como tal parece ser perfectamente aceptable. Queda abierta la cuestión del criterio de validez: no necesita preocuparnos aquí si se trata de una norma básica lógico-trascendental (Kelsen), una regla de reconocimiento (Hart), la constitución positiva (Engisch) o un soberano (Bentham, Austin).

La tesis II es una consecuencia lógica de I y también intuitivamente clara. Es indiferente si el orden jurídico es concebido como compuesto por las normas generales provenientes de la legislación (leyes) solamente, o si se le agregan también las normas provenientes de la costumbre y de la jurisprudencia, o si es considerado incluso como la totalidad de todas las normas generales e individuales. En todos los casos se trata de un conjunto (mayor o menor) de normas jurídicas. Cabe, pues, interpretar la tesis II como una definición de un concepto aún más general de orden jurídico.

La tesis III es proclamada a menudo por los juristas, pero no es claro qué entienden ellos por "sistema". Este término 
se va a emplear aquí en un sentido riguroso: un sistema (deductivo) es un conjunto de enunciados que contiene todas sus consecuencias (lógicas). ${ }^{4} \mathrm{Si}$ esta definición es aplicada a un orden jurídico resulta que un sistema jurídico es un conjunto de normas que contiene todas sus consecuencias. ${ }^{5}$ De ahí se sigue la necesidad de distinguir entre dos clases de normas: normas expresamente formuladas y normas que se derivan de aquellas (son sus consecuencias lógicas), que se llamarán normas derivadas. Estas últimas pertenecen también al sistema, aun cuando no hayan sido creadas expresamente por ningún legislador. ${ }^{\circ}$

La tesis IV expresa una verdad empírica que difícilmente podría ser puesta en duda, al menos en lo referente al derecho positivo. En todo caso, nos referiremos exclusivamente a este último. La legislación consiste precisamente en la creación y derogación de normas jurídicas positivas; la posibilidad de la legislación presupone ya la verdad de IV.

La tesis V es una consecuencia lógica de IV y ha de ser aceptada también, si se acepta IV.

La tesis VI parece muy plausible: probablemente es aceptada por casi todos los juristas. Sin embargo, es incompatible con II. Si el orden jurídico es concebido como un conjunto de normas, resulta claro que no puede cambiar sin perder su identidad. Cada vez que se crea una nueva norma o se deroga una norma perteneciente al orden, obtenemos un nuevo conjunto, distinto del conjunto originario. La identidad del conjunto está dada precisamente por la identidad de sus elementos. La distinción entre un orden jurídico (invariable) y su contenido (cambiante), expresada en la tesis VI, es incompatible con el concepto de orden jurídico como conjunto

4 Cfr. Alfred Tarski, Logic, Semantics, Metamathematics (Oxford, 1956), pp. 30 ss., 38 ss. y 60 ss.

5 Cfr. Alchourrón y Bulygin, Metodología, cap. IV, y Normative Systems (Viena-Nueva York, 1971), pp. 54 ss.

6 Cfr. G. H. von Wright, Norm and Action (London, 1963), pp. 156 ss.; traducción al castellano, Norma y Acción (Madrid, 1970). 
de normas. El orden jurídico como conjunto de normas es un orden momentáneo, es decir, referido a un determinado momento temporal. ${ }^{7}$ Por otra parte, tal terminología no coincide con los usos lingüísticos corrientes entre los juristas, lo cual sugiere la posibilidad de que haya más de un concepto de orden jurídico. Seguramente ha de haber un sentido de "orden jurídico" que haga verdadera la tesis VI. Pero tampoco es fácil abandonar la tesis II. Si se quiere mantener ambas tesis, ha de darse distinta interpretación a la expresión "orden jurídico": habría entonces dos conceptos de orden jurídico, encubiertos por la ambigüedad de esa expresión.

Una manera de conciliar las tesis II y VI es asignarle a "orden jurídico" el sentido no de un conjunto de normas, sino de una secuencia de conjuntos. La unidad de esta secuencia y con ello la identidad del orden juridico (en el sentido de VI) estaría dada por la identidad de los criterios usados para la identificación de los conjuntos normativos pertenecientes a la secuencia. ${ }^{8}$ La aplicación de los mismos criterios en tiempos diferentes lleva a resultados diferentes: esto se expresa diciendo que el contenido del orden es variable. Pero sólo si se modifican los criterios de identificación, es decir, son reemplazados por otros (como ocurre, por ejem. plo, en el caso de una revolución o una declaración de independencia) pierde el orden jurídico su identidad y es sustituido por otro orden nuevo.

Para evitar la ambigüedad de "orden jurídico" señalada más arriba, utilizaremos el término "sistema" para referirnos a conjuntos de normas y "orden" en el sentido de secuencia de conjuntos de normas. Un orden jurídico es, de acuerdo a esta convención, una secuencia de sistemas normativos.

La tesis VII - por más evidente que parezca - es incom. patible con las tesis III y IV, como se verá luego. Si se aceptan estas dos tesis, VII ha de ser rechazada.

7 Cfr. Joseph Raz, The Concept of a Legal System (Oxford, 1970), pp. 34 y ss. Raz fue el primero en señalar el carácter momentáneo del sistema jurídico.

8 Cfr. Alchourrón y Bulygin, Normative Systems, pp. 72 ss.; Metodología, pp. 118 s8. 
La tesis VIII es una consecuencia de VII y aparentemente no puede mantenerse, si VII resulta falsa.

Para decidir esta cuestión tenemos que analizar en detalle la estructura lógica de los actos por medio de los cuales son creadas o aniquiladas las normas jurídicas; los llamaremos actos de promulgación y actos de derogación. Dos preguntas nos van a interesar en especial: (1) ¿Qué se hace cuando se crea una norma? y (2) ¿Qué se hace cuando se deroga una norma?

\section{Promulgación de normas}

Nuestra primera pregunta, "¿Qué se hace cuando se crea una norma?", podría ser contestada diciendo que se agrega esa norma al conjunto de normas originario. Pero tal respuesta sería claramente insatisfactoria. Lo que se agrega al sistema en un acto de promulgación no es sólo la norma promulgada, sino muchas otras cosas.

Sea $\alpha$ un conjunto de normas formuladas; el conjunto de todas las consecuencias de $\alpha$ es un sistema normativo. Si mediante la promulgación se agrega a $\alpha$ una nueva norma $n$, obtenemos un nuevo sistema que se compone de la suma de los tres conjuntos siguientes: (a) las consecuencias de $\alpha$, (b) las consecuencias de $\{n\}$, y (c) las consecuencias de $\{\alpha+n\}$, que no sean derivables de $\alpha$ solamente, ni de $\{n\}$ solamente.

\section{Ejemplo 1}

Supongamos que $\alpha$ consiste en las dos normas siguientes: " $\mathrm{Si}$ la acción $p$ está permitida y la acción $q$ está permitida, entonces está permitida la acción $r$ " (en símbolos: $P p \cdot P q \supset P r$ ) y "Es obligatorio hacer $p$ " $\left(O_{p}\right)$. Supongamos, además, que se promulga una nueva norma "Es obligatorio hacer $q$ " $(O q)$. Al nuevo sistema pertenecen tanto las normas "La 
acción $p$ está permitida" $(P p)$, que es una consecuencia de $\alpha$, "La acción $q$ está permitida" $(P q)$, que es una consecuencia de la norma ' $O q$ ', como también la norma "La acción $r$ está permitida" $(P r)$ que no puede ser derivada ni de $\alpha$ solamente $\left(\sin { }^{\prime} O q^{\prime}\right)$, ni de ' $O q^{\prime}$ ' solamente $(\sin \alpha)$, pero sí de la suma de ambas. Las relaciones de deducción están representadas gráficamente (por medio de flechas) en el diagrama siguiente:

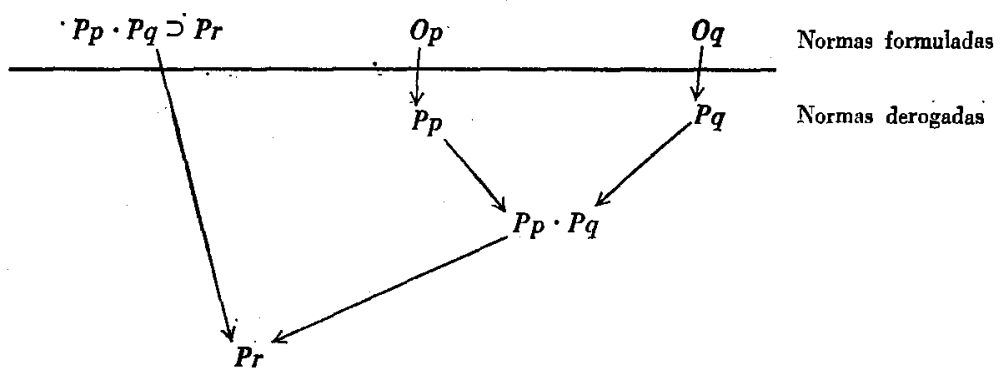

Por complicada que sea la situación, la promulgación de normas es siempre unívoca en el sentido de que el resultado de añadir una determinada norma a un determinado sistema normativo es un determinado sistema normativo.

\section{Derogación de normas}

Nuestra segunda pregunta era: “¿Qué se hace cuando se deroga una norma?" Aquí la situación es todavía más complicada y hay que tomar en cuenta diversas posibilidades. En primer lugar, puede tratarse de la derogación de una formulación de una norma, sin que se aniquile con ello ninguna norma del sistema. En segundo lugar, hay que distinguir entre la derogación de una norma formulada y la de una norma derivada. Todos estos casos serán examinados por separado.

\section{A. Derogación de una formulación de norma}

Cuando en un sistema normativo una norma ha sido formu- 
lada dos veces, es decir, cuando hay dos formulaciones que expresan la misma norma, o cuando se formula expresamente una norma, que ya pertenecía al sistema como norma derivada, entonces el sistema resulta redundante respecto de esa norma y la formulación redundante puede ser eliminada, sin que el sistema se modifique. Este es un caso bastante frecuente en la práctica legislativa; tal operación es también llamada derogación, pero no se trata aquí de la derogación de una norma, sino tan sólo de la derogación de una formulación: una expresión lingüística es eliminada de un texto, pero no se elimina ninguna norma del sistema. La norma expresada por el texto derogado no se deroga; permanece en el sistema, aun cuando a veces sólo como norma derivada.

\section{Ejemplo 2}

Las normas "Es obligatorio hacer $p$ y $q$ " $(O(p \cdot q))$ y "La acción $p$ está permitida" $(P p)$ pertenecen a las normas formuladas del sistema. Si se deroga la norma ' $P p$ ', el sistema permanece invariable, pues las consecuencias de $\{O(p \cdot q), P p\}$ son las mismas que las consecuencias de $\{O(p \cdot q)\}$. Lo único que ha cambiado aquí es la presentación del sistema: la norma ' $P p$ ', ya no pertenece más a las normas formuladas, sino a las derivadas. Es evidente que este tipo de "derogación" es unívoco.

\section{B. Derogación de una norma formulada}

Este puede ser considerado como el caso "normal" de derogación. Aquí se deroga una determinada norma, para colocar, eventualmente, en su lugar otra norma diferente. Lo que se deroga aquí no es, sin embargo, únicamente la norma expresamente derogada, sino también todas las normas que se derivan de ella y también las normas para cuya derivación ella es necesaria. 


\section{Ejemplo 3}

El sistema se compone de las normas ' $P p \cdot P q \supset P r$ ', ' $O p$ ' y ' $O q$ ', y se quiere derogar la norma ' $O q$ '. La derogación de ' $O q$ ' implica derogar también las normas ' $P q^{\prime},{ }^{\prime} P p \cdot P q$ ' y 'Pr'.

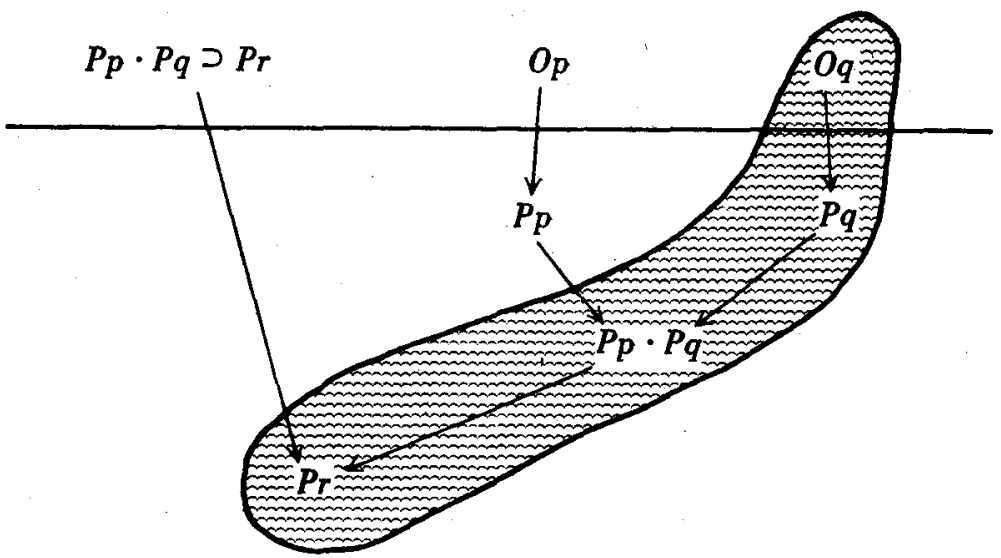

La zona sombreada simboliza el ámbito de la derogación: todas las normas que se hallan en ella quedan afectadas por la derogación de ' $O q$ ' y son eliminadas del sistema en el mismo acto en que es derogada ' $O q$ '. No se trata aquí, pues, de una relación temporal; sería erróneo pensar que primero se deroga ' $O q$ ' y luego se eliminan las otras normas. La situa. ción es otra: el acto de derogar ' $O q$ ' implica lógicamente que las otras normas están derogadas. La derogación de las consecuencias de la norma derogada forma parte del contenido del acto de derogación.

También en este caso la derogación es unívoca: la eliminación de una norma determinada de un sistema determinado conduce a otro sistema, también determinado.

En los casos considerados bajo 3.A. y 3.B. se trata de dos tipos de derogación, o mejor, de dos conceptos de derogación. 
En 3.A. se elimina una formulación, pero el contenido conceptual del sistema permanece igual. En 3.B. se elimina tanto una formulación como una norma; el acto de derogar provoca un cambio del sistema: después de la derogación tenemos un nuevo sistema, distinto del anterior (aun cuando ambos pertenezcan a la misma secuencia, es decir, al mismo orden jurídico). En el caso 3.C. que analizaremos a continuación se trata del mismo concepto de derogación que en 3.B. La diferencia está tan sólo en que primariamente se trata de una modificación del contenido conceptual del sistema, pero a consecuencia de ésta la formulación del sistema ha de ser también modificada.

\section{C. Derogación de una norma derivada}

Cuando lo que se deroga es una norma derivada, no formulada, pueden originarse situaciones muy extrañas. Vamos a considerar dos ejemplos: en el primero, la derogación de una norma derivada conduce a un resultado unívoco, pero en el segundo el resultado no es un sistema, sino una pluralidad de sistemas alternativos.

\section{Ejemplo 4}

En el sistema consistente de las normas ' $P p \cdot P q \supset P r^{\prime}$, ' $O p$ ' y ' $O q$ ' ha de ser derogada la norma ' $P q$ ', por ejemplo, el legislador dicta una norma declarando que la acción $q$ no ha de considerarse como permitida. ¿Qué normas quedan afectadas por este acto de derogación?

Es claro que la norma ' $O q$ ' que implica a la norma derogada, también debe considerarse como derogada, pues de lo contrario tampoco se habría derogado ' $P q^{\prime}$ que podría ser fácilmente derivada de ' $O q$ '. Pero también las normas derivadas con la ayuda de ' $P q^{\prime}-{ }^{\prime} P p \cdot P q$ y ' $P r$ ' - deben ser eli- 
minadas, pues $\sin$ ' $P q^{\prime}$ ' ellas ya no pueden derivarse. ${ }^{\ominus}$ Resulta, pues, que la derogación se extiende tanto "hacia arriba", es decir, a las normas implicantes, como también "hacia abajo", esto es, a las normas implicadas. En el diagrama siguiente la zona sombreada simboliza el ámbito de la derogación de la norma ' $P q$ '.

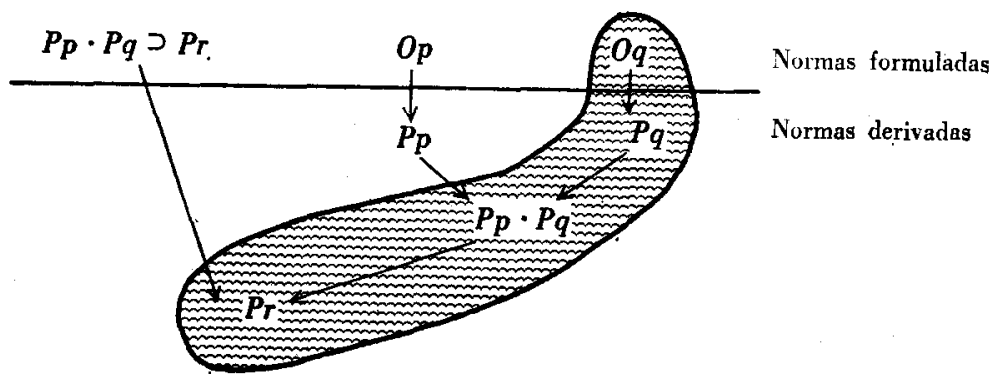

En este caso la derogación de la norma derivada ' $P q$ ' equivale a la derogación de la norma formulada ' $O q$ '. Pero no siempre ocurre esto.

\section{Ejemplo 5}

Supongamos que en el sistema anterior se quiera derogar la norma ' $P p \cdot P q$ '. La derogación de esta norma implica la derogación de la norma 'Pr' que deriva de ella. Pero ¿qué ocurre con las normas ' $O p$ ' y ' $O q$ '? Es evidente que por lo menos una de estas normas debe desaparecer, pero la pregunta es ¿cuál de ellas? No hay criterios lógicos para decidir esta cuestión; se trata de un problema insoluble. El resultado de la derogación de la norma ' $P p \cdot P q$ ' es, por lo tanto, no

${ }^{9}$ En realidad no siempre es así; la indeterminación puede extenderse eventualmente hacia las normas implicadas. Pero no vamos a analizar esta complicación. 
un sistema normativo, sino por lo menos dos sistemas alternativos, que se representan en el diagrama siguiente:
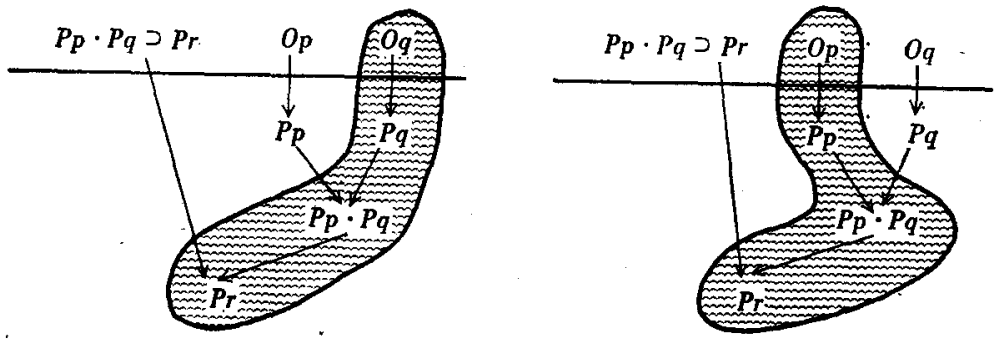

También este tipo de derogación aparece con frecuencia en la práctica legislativa. El ejemplo típico y más corriente es la cláusula que suele insertarse al final de una ley, declarando que "quedan derogadas todas las disposiciones que se oponen a la presente ...". Tal disposición puede, sin em. bargo, destruir la unidad del sistema, fracturándolo en diversas alternativas. Los juristas parecen no haber reparado en esta consecuencia, ciertamente desastrosa, omisión que se debe probablemente al hecho de que en la mayoría de los casos se decide por una de las alternativas posibles sobre la base de consideraciones extralógicas. Casos en los que las alternativas no sólo sean lógicamente equivalentes, sino tam. bién igualmente razonables desde el punto de vista de la justicia o de sus consecuencias prácticas, son relativamente poco frecuentes. ${ }^{10}$

El último caso analizado (derogación de una norma derivada) muestra claramente que las dos operaciones - creación y derogación de las normas- no son simétricas. Nada

10 Es interesante observar que en las Directivas para la legislación de la cancillería austriaca (Legistische Richtlinien des Bundeskanzleramtes, 1970, 43.984-2b/70) se recomienda expresamente evitar la derogación indeterminada (derogación de todas las disposiciones que se oponen a una norma dada) y sólo usar la derogación formal (explícita) -directivas 23 y 24- pero no se aduce fundamento alguno para esa exigencia. 
análogo a la indeterminación lógica del sistema puede producirse al crearse una nueva norma.

En lo sucesivo se va a usar la expresión "indeterminación lógica del sistema normativo" como término técnico para caracterizar la situación en la que en lugar de un sistema aparecen varios sistemas alternativos.

La posibilidad de la indeterminación lógica prueba que la opinión tan difundida entre los juristas que hemos sintetiza. do en la tesis VII, de acuerdo a la cual es siempre posible decidir qué normas pertenecen en un momento dado a un sistema normativo determinado, debe ser calificada como errónea. Esta cuestión no siempre es decidible y cabe subrayar, además, que la indeterminación lógica aparece con mucha mayor frecuencia de lo que podría pensarse a primera vista. Tal situación puede (aunque no necesariamente) darse cada vez que el legislador emplea una cláusula general que deroga todas las normas que se oponen a una norma dada, sin especificar cuáles son todas esas normas.

Ya hemos visto que el postulado de la existencia continua del orden jurídico (tesis VI) sólo puede mantenerse si el orden jurídico es concebido como una secuencia de sistemas jurídicos (conjuntos de normas). Los sistemas jurídicos no tienen existencia continua, pues son relativos a un momento temporal dado. Pero este tiempo tiene propiedades peculiares: se trata de un tiempo discontinuo, discreto, y los momentos temporales están dados por los actos de promulgación y derogación de las normas, es decir, básicamente por los actos legislativos. Todo acto que crea una nueva norma o deroga una norma existente determina un momento nuevo y con ello un nuevo sistema. (Naturalmente puede haber actos legislativos que no producen modificación alguna del sistema, como por ejemplo, cuando se deroga o se crea una norma redundante.)

También el postulado de la unicidad del orden jurídico (tesis VIII) se vuelve ahora problemático. En relación a los sistemas de normas es seguramente falso; a lo sumo puede valer para el orden jurídico como secuencia de sistemas. Pero 
la posibilidad de la indeterminación lógica implica que esta secuencia no siempre es lineal, pues a veces se ramifica. Cuando esto ocurre, la unidad del sistema sólo puede ser restablecida mediante la reformulación del sistema, al establecer expresamente cuáles son las normas que pertenecen al sistema y cuáles no. Es decir, se decide por una de las alternativas posibles. Esta actividad es creadora y en principio incumbe al legislador, aun cuando a menudo sea llevada a cabo por la ciencia del derecho.

\section{Lagunas, contradicciones y la indeterminación lógica}

Un sistema jurídico puede, como se sabe, ser defectuoso por razones de fondo: puede contener prescripciones injustas, poco razonables o por algún motivo inconvenientes. Pero también pueden presentarse defectos puramente formales o lógicos. La falta de completitud, la incoherencia y la indeterminación lógica pertenecen a estos últimos.

El problema de la completitud del sistema jurídico es conocido en la literatura jurídica como el problema de las lagunas del derecho. Cuando una acción no está regulada por el sistema jurídico, se dice que hay una laguna; un sistema con lagunas es incompleto.

El problema de las lagunas del derecho es complejo y equívoco. En realidad son varios problemas distintos que reciben este rótulo en la tradición jurídica. ${ }^{11}$ Aquí nos contentaremos con mencionar algunos aspectos del problema. El concepto de laguna normativa puede ser definido en términos de la determinación normativa. Una acción $p$ (perteneciente a una determinada clase de acciones $H$ ) está normativamente determinada en el sistema $S$ si, y sólo si, $p$ está permitida o prohibida en $S$, esto es, si una norma de la forma ' $P p$ ' u ' $O-p$ ' pertenece a $S .{ }^{12}$ Cuando una de las acciones de la clase $H$ no está determinada en $S$, tenemos una laguna y el sistema

11 Cfr. Alchourrón y Bulygin, Metodologia.

12 Tales normas no tienen porque ser formuladas explícitamente; basta con que una de ellas se derive del sistema. 
$S$ es incompleto respecto de $H$. Sólo cuando todas las acciones de $H$ están determinadas, el sistema $S$ es completo en relación a $H$. La completitud (laguna) es, por lo tanto, un concepto relacional; es una relación entre un sistema de normas y una clase de acciones y no una propiedad (absoluta) del sistema, cosa que es a menudo pasada por alto por los juristas. Tratándose de sistemas jurídicos que por lo común contienen normas condicionales (hipotéticas), tenemos una relación triádica: entre un sistema de normas, una clase de acciones y una clase de circunstancias fácticas o casos. ${ }^{18} \mathrm{De}$ ahí se sigue que las preguntas de la forma " ¿Es el orden juridico $S$ (o el derecho a secas) completo o tiene lagunas?", tal como se formulan a menudo por los juristas y los filósofos del derecho, carecen de respuesta, mientras no se determinen los ámbitos de los tres argumentos de la relación. El análisis de la estructura lógica (sintáctica) del concepto de completitud -como de los conceptos jurídicos en general- es una tarea importante de la filosofía jurídica, que sólo puede ser llevada a cabo con la ayuda de la lógica moderna. ${ }^{14}$

En este contexto cabe señalar, además, que los juristas confunden con frecuencia la ausencia de una regulación con una regulación de alguna manera insatisfactoria (conforme a algún criterio valorativo).$^{15}$ Esta última situación suele ser también calificada como 'laguna'; algunos autores llegan a sostener que sólo puede haber lagunas en este último sentido. ${ }^{18}$ Tal terminología no es aconsejable, pues conduce a confusión: una regulación deficiente es una regulación y no ha de ser confundida con la ausencia de toda regulación.

Una confusión similar puede producirse cuando se habla de lagunas para referirse a la dificultad de la subsunción

13 Cfr. Alchourrón y Bulygin, Metodología, donde se usan las expresiones "universo de casos" y "universo de acciones" como términos técnicos.

${ }_{14}$ Cfr. H. Wagner y K. Haag, Die moderne Logik in der Rechtswissenschaft (Bad Homburg, 1970), pp. 20-23.

15 Cfr. Alchourrón y Bulygin, Normative Systems, pp. $106-116$ y Metodolo. gía, pp. 145 ss.

16 Hans Kelsen, Reine Rechtslehre, 2a. edición (Viena, 1960), pp. 251 ss. 
causada por la indeterminación semántica (vaguedad) de los conceptos, como lo hace, por ejemplo, Kantorowicz al afirmar que en la ley hay tantas lagunas como palabras. ${ }^{17}$ Se trata aquí de un problema totalmente distinto. La aplicación de una norma general a una situación concreta puede dar lugar a dificultades cuyo origen ha de buscarse en las propiedades semánticas del lenguaje (vaguedad actual o potencial de los conceptos). Ya Heck decía que los conceptos no están delimitados con claridad; el núcleo conceptual (Begriffskern) está rodeado por un halo (Begriffshof); cuando una situación concreta cae dentro del ámbito del 'halo', no se sabe si la norma es aplicable o no. ${ }^{18}$ Se trata, pues, de un problema gnoseológico. La filosofía del lenguaje moderna se ha ocupado intensamente de estos temas, si bien los resultados alcanzados no siempre son debidamente tomados en cuenta por los juristas. ${ }^{10}$

Llenar las lagunas es un problema práctico importante. Los juristas utilizan para ese fin toda una serie de reglas, que aparecen bajo la forma de reglas de inferencia: el argumento a fortiori, el argumento a contrario, el argumento analógico, etc. Es importante tener presente que ninguna de tales reglas es una regla de inferencia lógicamente válida; sólo con la ayuda de premisas implícitas pueden llevar a conclusiones válidas. ${ }^{20}$ Con medios puramente lógicos no se puede completar un sistema incompleto.

La contradicción entre normas origina un problema distinto, aunque similar en sus efectos prácticos. Una laguna

17 H. Kantorowicz, "La lucha por la ciencia del derecho" en La Ciencia del Derecho (autores varios) (Buenos Aires: Losada, 1949), pp. 323-371. El término "laguna" es usado en un sentido similar por H. L. A. Hart en Derecho y Moral (Buenos Aires, 1962) y G. R. Carrió en Notas sobre Derecho y Lenguaje (Buenos Aires, 1965), pp. 47 y ss.

18 Philipp Heck, Begriffsbildung und Interessenjurisprudenz (Tübingen, 1932), pp. 52-53.

19 La primera exposición sistemática de estos problemas fue realizada entre nosotros por Genaro R. Carrió, op. cit. Véase también Carlos S. Nino, Notas de Introducción al Derecho, 4 volúmenes (especialmente vol. 4) (Buenos Aires, 1975).

${ }^{20}$ Cfr. C. E. Alchourrón, "Argumentos jurídicos a fortiori y a pari", Revista Juridica de Buenos Aires, IV, 1961, pp. 177-199. 
es una situación no regulada; el sistema contiene demasiado pocas normas y la dificultad sólo puede ser superada mediante el agregado de una nueva norma al sistema. En cambio, en el caso de contradicción tenemos una superabundancia de normas: el sistema contiene demasiadas normas y la solución del problema exige la eliminación de por lo menos una de las normas contradictorias. Las dos situaciones son, sin embargo, parecidas en el sentido de que sus consecuencias prácticas son similares: en ambos casos el sistema fracasa en su función más importante, la de regular la conducta social. En ambos casos el sistema está determinado. Se sabe muy bien cuáles son las prescripciones del sistema (en esto se diferencian tanto las lagunas como los conflictos normativos de la indeterminación lógica), pero no se puede ajustar su conducta a lo que el sistema prescribe: en el caso de la laguna, porque no hay ninguna conducta que esté conforme, ni tampoco contraria al sistema; en el caso de la contradicción, porque toda conducta es a la vez conforme y contraria al sistema. En ambos casos se trata de un problema lógico, no meramente gnoseológico. No es que simplemente no se sepa cuál es la conducta requerida por el sistema; el sistema no lo determina.

La definición de la contradicción normativa, es decir, de la incompatibilidad formal entre las normas no carece de dificultades. No es fácil encontrar un criterio intuitivamente aceptable y al mismo tiempo adecuado desde el punto de vista formal. ${ }^{21}$ Lo más conveniente parece ser la definición en términos del cumplimiento de la norma: dos normas son contradictorias cuando el cumplimiento de una de ellas excluye, por razones lógicas, el cumplimiento de la otra. La obligación y la prohibición simultáneas de una acción ofrecen el ejemplo intuitivamente más claro de la incompatibi-

21 Cfr. G. H. von Wright, Norm and Action, pp. 134 ss., y también C. E. Alchourrón y E. Bulygin, "Von Wright on Deontic Logic and the Philosophy of Law" en P. A. Schilpp (editor), The Philosophy of Georg Henrik von Wright (La Salle, Illinois: Library of Living Philosophers, en prensa). 
lidad formal. De acuerdo con este criterio las normas ' $O p$ ' y ' $O-p$ ' son contradictorias.

Este criterio no puede, sin embargo, ser aplicado directamente a las normas permisivas, ya que aquí no cabe hablar del cumplimiento o la violación de la norma. De las permi. siones se puede hacer uso, pero no se las puede obedecer ni violar. Cabe, sin embargo, extender el criterio a las normas permisivas, estableciendo que una prohibición (o una obligación) está en contradicción con una permisión, cuando el uso de la permisión hace lógicamente imposible el cumplimiento de la prohibición (obligación) y viceversa. De acuerdo con esto, las normas ' $P p^{\prime}$ y ' $O-p$ ' resultan contra. dictorias. Dos normas permisivas son, en cambio, siempre compatibles.

La eliminación de las contradicciones, como el llenado de las lagunas, no es un problema lógico. La lógica sólo puede ayudar a constatar la presencia de los conflictos normativos, pero no puede eliminarlos. Las reglas usadas por los juristas para ese fin (lex posterior, lex superior, lex specialis) son prácticamente útiles, pero no son lógicamente válidas. También aquí se trata de un problema creador del derecho (en un sentido negativo) que compete en primer lugar al legislador.

La situación es también similar en el caso de la indeterminación lógica, en lo que a las consecuencias prácticas se refiere, aunque su origen sea totalmente distinto. También aquí fracasa el sistema, porque la conducta no puede conformarse a él, pero no porque haya demasiadas o demasiado pocas normas, sino porque hay demasiados sistemas y no hay criterios lógicos para elegir una de las alternativas posibles.

$\mathrm{El}$ análisis de la estructura lógica de los actos legislativos (promulgación y derogación) nos obliga a revisar los conceptos tradicionales de orden jurídico y sistema jurídico y señala ciertos peligros que pueden presentarse con la derogación apresurada ý de esta manera muestra la utilidad práctica del análisis lógico de los conceptos jurídicos. 
An idea widely recognized and accepted by jurists is that legal norms form an order. The concept of legal order requires, consequently, an analysis based on the notion used by jurists. In this paper, legislative and not customary norms are taken into account. Two terminological explanations should be made at this point: (1) norms rather than propositions about norms are discussed, and (2) "legal norm" should be interpreted in its broadest sense, that is, statements that form part of legal texts, whether they be normative or not.

The intuitive notion of legal order can be said to consist of eight theses:

I. It is the totality of valid norms according to a criterion of validity.

II. It is a set of norms.

III. It is a system of norms.

IV. Its norms are created and derogated by acts of will.

V. It is subject to changes in time.

VI. The changes affect its content but not its identity.

VII. Its content is, in principle, determinable at all times.

VIII. In each State there is one and only one legal order.

Although these theses are intuitively acceptable, it is not easy to mold them into one coherent theory, since several of them are con. tradictory (III and IV with VII and VIII).

Theses I and II are acceptable, although incomplete, definitions of "legal order" which could be made more precise.

With regard to III, "system" is understood to be a deductive system - a set of sentences which contains all its logical consequences- and a distinction is made, therefore, between norms that are expressly formulated and those that are derived. Theses IV and V are acceptable almost without doubt, especially as they refer to positive law. Thesis VI, accepted by almost all jurists, is, nevertheless, incompatible with II. If a legal order is a set, it cannot change without losing its identity since the identity of a set depends on that of its elements. The solution would be to recognize two concepts of legal order covered by the same expression, or to submit that "legal order" designates a sequence of sets, and that only a modification of the identification criteria would make it lose its identity. 
The article deals with the alleged contradictions of III and IV with VII and VIII, presenting first the problem that if VII is false, then VIII is also false because the latter is a consequence of the former. For this reason, the notion of "promulgation of norms" is explained. It is shown, by means of an example, that the promul. gation adds not only a promulgated norm, but also its logical consequences as well as the derivables of the union of such norms with the system. This promulgation is always univocal because it produces a definite normative system.

In the explanation of the notion of "derogation of norms", mention is made of the derogation of the formulation of a norm, which eliminates not a norm but a redundant formula. Exemplification is also given of the derogation of a formulated norm. Here also not only the norm but also all its consequences are derogated, and it is univocal: it leads to another definite system. Finally, the derogation of a derived norm is discussed, and it is shown, with examples, that it is possible that this derogation may lead to a plurality of alternative systems. This shows that creation and derogation are not symmetrical since the latter can produce a logical indetermination of the system, that is, the situation in which various alternative systems appear. With this, the falseness of VII is proved, and therefore VI and VIII become problematic. If III and IV are accepted, thesis VII must be rejected.

In Section 4, the logical defects of legal systems are dealt with, as are logical indetermination, gaps, and contradictions, with mention made to some aspects of these problems.

Regarding the gaps, some of the ideas contained in the authors' book, Normative Systems, are put forth. Later, a definition of "normative contradiction" is offered, with recognition of the fact that it is difficult to satisfy the formal point of view and intuitions at the same time. It is contended that two norms are contradictory when the fulfilment of one of them excludes, for logical reasons, the fulfilment of the other. Logical indetermination has, at the same time, the same practical consequences, although its origin is different. Logic alone cannot solve any of these problems, but it allows that they be made known and permits the examination of certain legal concepts whose analysis has practical application.

(Summary by Javier Esquivel) 